\title{
Aryl hydrocarbon receptor inhibits inflammation in DSS-induced colitis via the MK2/p-MK2/TTP pathway
}

\author{
QIMENG WANG, KUNQIU YANG, BIN HAN, BAIFA SHENG, JIUHENG YIN, AIMIN PU, \\ LIANGZI LI, LIHUA SUN, MIN YU, YUAN QIU, WEIDONG XIAO and HUA YANG
}

\begin{abstract}
Department of General Surgery, Xinqiao Hospital, Third Military Medical University, Chongqing 400037, P.R. China
\end{abstract}
Received January 29, 2016; Accepted November 9, 2017

DOI: $10.3892 /$ ijmm.2017.3262

\begin{abstract}
The pathogenesis of inflammatory bowel disease (IBD) is believed to be associated with the abnormal expression of inflammatory factors. The aryl hydrocarbon receptor $(\mathrm{AhR})$ is a ligand-dependent transcription factor, which can suppress the inflammatory response and attenuate experimental colitis. However, the detailed mechanism underlying the effects of AhR remains unclear. The present study investigated the role of AhR in the pathogenesis of IBD. Colitis was induced in mice by administration of 3\% dextran sulphate sodium (DSS) for 7 days. The mice were also administered injections of the AhR agonist, 6-formylindolo(3,2-b)carbazole (FICZ), starting 2 days after the first administration of DSS. Furthermore, LoVo cells were treated with lipopolysaccharide (LPS) in the presence or absence of FICZ for $8 \mathrm{~h}$. The protein expression levels of AhR, cytochrome P450 1A1 (CYP1A1) and tristetraprolin (TTP) were assessed by western blotting and immunofluorescence, whereas mRNA expression levels were assessed by reverse transcription-quantitative polymerase chain reaction. The results indicated that injection of mice with FICZ significantly attenuated DSS-induced colitis; in addition, the expression levels of inflammatory cytokines were markedly downregulated. Conversely, the expression levels of AhR and TTP were upregulated. In addition, mice in the AhR-knockout + DSS group exhibited elevated inflammatory cytokine production and developed more severe colitis. In LoVo cells, incubation with FICZ decreased the expression levels of inflammatory cytokines, whereas AhR and TTP expression was increased. In addition, the levels of phosphorylated-mitogen-activated protein kinase-activated protein kinase 2 (p-MK2) were decreased. These results suggested that AhR deficiency resulted in increased susceptibility to colitis, whereas activation of AhR by FICZ could ameliorate DSS-induced colitis via the MK2/p-MK2/TTP pathway.
\end{abstract}

Correspondence to: Mr. Hua Yang, Department of General Surgery, Xinqiao Hospital, Third Military Medical University, 83 Xinqiao Main Street, Chongqing 400037, P.R. China

E-mail: huayang@tmmu.edu.cn

Key words: inflammatory bowel disease, 6-formylindolo(3,2-b) carbazole, aryl hydrocarbon receptor, tristetraprolin, inflammation

\section{Introduction}

The pathogenesis of inflammatory bowel disease (IBD) in humans remains incompletely understood; however, considerable evidence indicates that IBD results from an interaction between genetic, immune and environmental factors $(1,2)$. IBD, including ulcerative colitis (UC) and Crohn's disease (CD), is a prominent intestinal disease. The pathogenesis of IBD is predominantly associated with intestinal immunodeficiency; this dysregulated immune response results in chronic gut inflammation and the presence of activated immune cells, which produce copious amounts of interferon (IFN)- $\gamma$, tumor necrosis factor (TNF)- $\alpha$, interleukin (IL)-2 and IL-17, thereby promoting the production of large amounts of inflammatory cytokines, and reactive oxygen and nitrogen metabolites, which ultimately lead to organ damage (3-5). Consistent with these findings, restoration of the balance between inflammatory and anti-inflammatory factors has reported as a useful treatment strategy, which has been successfully tested in patients with IBD and in experimental animal models of colitis (6-9).

Previous studies have reported that the aryl hydrocarbon receptor $(\mathrm{AhR})$ is a transcription factor, which serves a protective role in IBD (10-12). AhR is extensively expressed in vertebrate cells and is a member of the basic helix-loop-helix/Per-Arnt-Sim homology superfamily (13). In the cytosol, AhR is present in an inactive form that binds to numerous co-chaperones (14). Following ligand binding, AhR dissociates from its chaperones and dimerises with AhR nuclear translocator (ARNT). The AhR/ARNT complex then activates the transcription of target genes, including cytochrome P450 1A1 (CYP1A1) (15). AhR has also been revealed to be indirectly activated by endogenous AhR ligands, including 6-formylindolo(3,2-b)carbazole (FICZ) (16). FICZ may activate AhR and significantly inhibit the inflammatory response (17). A previous study in AhR-knockout (KO) mice illustrated the role of $\mathrm{AhR}$ in the function and development of various organs; skin defects and a spectrum of hepatic abnormalities, as well as haematopoietic and vascular abnormalities, were observed in AhR-KO mice (18). Therefore, reduced AhR expression may lead to immunodeficiency and cause the immune system to secrete a mass of inflammatory factors. Although numerous studies have demonstrated a role for AhR in the inhibition of inflammation, the detailed underlying mechanism remains unclear. 
It has previously been reported that treatment of tristetraprolin (TTP)-KO mice with dextran sulphate sodium (DSS) leads to increased susceptibility to colitis, decreased colon length and increased production of proinflammatory cytokines (19). These findings suggest that TTP deficiency may result in a defect in the resolution of intestinal inflammation and in exacerbated IBD symptoms. TTP is the prototypic member of the TIS11 family of RNA-binding proteins, which has an important role in regulating the expression of adenylate-uridylate-rich element (ARE)-containing mRNAs (20). RNA-binding proteins, including TTP and human antigen $\mathrm{R}(\mathrm{HuR})$, recognise and bind to AREs in the 3'-untranslated regions (3'-UTRs) of their target mRNAs $(20,21)$. Binding of TTP to its target mRNAs promotes the degradation of numerous inflammatory factors, including TNF- $\alpha$, IFN- $\gamma$, IL-1 $\beta$, IL-6, IL- 8 and cyclooxygenase (COX)-2 (22-24). Furthermore, TTP-KO mice display severe autoimmune dysfunction, inflammatory arthritis and ulcerative colitis, thus suggesting that TTP has an important role in limiting the inflammatory response (25).

A previous study indicated that the expression of TTP is downregulated in $\mathrm{AhR}^{-/-}$cells (26). Furthermore, AhR is able to suppress the expression of COX-2, which is mediated by HuR, another RNA-binding protein from the same family as TTP (27). Based on the results of previous studies, the present study hypothesised that AhR may exert protective effects against experimental colitis through the regulation of TTP expression.

\section{Materials and methods}

Materials. FICZ was purchased from Enzo Life Sciences, Inc. (Farmingdale, NY, USA). Lipopolysaccharide (LPS) was purchased from Sigma-Aldrich (Merck KGaA, Darmstadt, Germany). Foetal bovine serum (FBS) and Dulbecco's modified Eagle's medium (DMEM) were purchased from HyClone (GE Healthcare Life Sciences, Logan, UT, USA). Antibodies against AhR (cat. no. ab2770) were purchased from Abcam, Inc. (Cambridge, MA, USA). Antibodies against CYP1A1 (cat. no. 13241-1-AP) and mitogen-activated protein kinase-activated protein kinase 2 (MK2; cat. no. 13949-1-AP) were purchased from Wuhan Sanying Biotechnology (Wuhan, China). Antibodies against TTP (cat. no. sc-14030) were purchased from Santa Cruz Biotechnology, Inc. (Dallas, TX, USA). Antibodies against phosphorylated (p)-MK2 were purchased from Cell Signalling Technology, Inc. (Danvers, MA, USA). Antibodies against GAPDH (cat. no. 10494-1-AP) were purchased from Wuhan Sanying Biotechnology. Goat anti-mouse (cat. no. A0216) and goat anti-rabbit (cat. no. A0208) horseradish peroxidase (HRP)-conjugated secondary antibodies, Cy3-conjugated goat anti-mouse immunoglobulin (Ig)G (cat. no. P0193) and fluorescein isothiocyanate-conjugated goat anti-rabbit IgG (cat. no. P0186) were purchased from Beyotime Institute of Biotechnology (Shanghai, China). All primers used in the present study were synthesised by Invitrogen (Thermo Fisher Scientific, Inc., Waltham, MA, USA).

Cell cultures. LoVo human intestinal epithelial cells were purchased from the American Type Culture Collection (Manassas, VA, USA) and maintained in DMEM supplemented with $15 \% \mathrm{FBS}, 100 \mathrm{U} / \mathrm{ml}$ streptomycin and $100 \mathrm{U} / \mathrm{ml}$ penicillin at $37^{\circ} \mathrm{C}$ in a $5 \% \mathrm{CO}_{2}$ atmosphere. The medium was refreshed every 2 days. The cells were subcultured successively when they reached $\sim 80 \%$ confluence.

Cell treatments. Cells were grown in 6-well plates and were incubated with LPS $(10 \mu \mathrm{g} / \mathrm{ml})$ and/or FICZ $(100 \mathrm{nM})$ for $8 \mathrm{~h}$ at $37^{\circ} \mathrm{C}$. Immunofluorescence and western blotting were used to detect target protein expression, and reverse transcription-quantitative polymerase chain reaction (RT-qPCR) was used to determine mRNA expression.

Small interfering (si)RNA transfection. For transient knockdown of AhR expression, cells were grown in DMEM with no antibiotics until they reached $80-100 \%$ confluence. Transient transfection was performed using Lipofectamine ${ }^{\circledR} 2000$ (Invitrogen; Thermo Fisher Scientific, Inc.) according to the manufacturer's protocol. After a 48-h transfection period, the cells were harvested. The sequences of the AhR-targeting siRNA were: Sense, 5'-GGAACACCUACAUCUAGAAdTdT-3' and antisense, 3'-dTdT CCUUGUGGAUGUAGAUCUU-5'. The sequences of the negative control siRNA were: Sense, 5'-GGGCAAAUCCCAAGAGGAAdTdT-3' and antisense, 3'-dTdT CCCGUUUAGGGUUCUCCUU-5'.

DSS-induced colitis. The present study was approved by the Laboratory Animal Welfare and Ethic Committee of the Third Military Medical University (Chongqing, China). Male C57BL/6J wild-type mice ( $\mathrm{n}=60$; age, 6-8 weeks; weight, 18-22 g) and male C57BL/6J AhR-KO mice ( $\mathrm{n}=15$; age, 6-8 weeks; weight, 18-22 g) were purchased from the Experimental Animal Center at Daping Hospital of the Third Military Medical University (Chongqing, China). Animals were bred and maintained under specific pathogen-free conditions in a temperature-controlled room $\left(20 \pm 2^{\circ} \mathrm{C}\right)$ with circadian light-dark cycles and free access to standard rodent chow and water. Mice were randomly divided into the following three groups: Sham $(n=10)$, DSS $(n=10)$, DSS + FICZ $(n=10)$ group. Colitis was induced by administration of $3 \%$ DSS (Sigma-Aldrich, Inc.; Merck KGaA) dissolved in distilled water for 7 days. Mice in Sham group were administered distilled water. In addition, FICZ ( $1 \mu \mathrm{g} /$ mouse) was administered daily, by intraperitoneal injection, beginning 2 days after the start of DSS administration. Changes in body weight were recorded daily. Following DSS administration for 7 days, the mice were sacrificed and intestinal mucosa samples were collected for histology, and the determination of target mRNA and protein expression levels.

Histological examination. Colon segments were washed three times in PBS and were fixed in $4 \%$ paraformaldehyde overnight at $4^{\circ} \mathrm{C}$. Ethanol was used to dehydrate the tissues, which were embedded in paraffin. The resulting tissue sections $(4-\mu \mathrm{m}$ thick) were stained with haematoxylin for $5 \mathrm{~min}$ and eosin for $3 \mathrm{~min}$ at room temperature. The sections were observed using a confocal microscope (LSM 5 PASCAL; Carl Zeiss, Oberkochen, Germany).

Immunofluorescence staining. Cells were fixed with 4\% paraformaldehyde for $15 \mathrm{~min}$ at room temperature, and permeabilized with $0.2 \%$ Triton $\mathrm{X}-100$ for $10 \mathrm{~min}$ at room temperature. Cells were then blocked in 5\% bovine serum albumin (BSA) (Sigma-Aldrich, Inc.; Merck $\mathrm{KGaA}$ ) for $2 \mathrm{~h}$ at $37^{\circ} \mathrm{C}$ and incubated with mouse monoclonal anti-AhR (1:50) and rabbit polyclonal anti-TTP 
Table I. Sequences of primers used in reverse transcription-quantitative polymerase chain reaction experiments.

\begin{tabular}{|c|c|c|c|}
\hline Species & Gene name & Forward (5'-3') & Reverse (5'-3') \\
\hline \multicolumn{4}{|l|}{ Human } \\
\hline & IL-1 $\beta$ & CTTCGACACATGGGATAACG & ATATCCTGTCCCTGGAGGTG \\
\hline & IL-6 & GACAGCCACTCACCTCTTCA & ССТСТTTGCTGCTTTCACAC \\
\hline & CYP1A1 & CCATGTCGGCCACGGAGTT & ACAGTGCCAGGTGCGGCTT \\
\hline & TTP & TTTAAGGGAGGCAATGAACC & CAGGAGACACTGGAACCTCA \\
\hline & $\beta$-actin & CCACGAAACTACCTTCAACTCC & CGTGATCTCCTTCTGCATCCTG \\
\hline \multicolumn{4}{|l|}{ Mouse } \\
\hline & IL-1 $\beta$ & GAAATGCCACCTTTTGACAGTG & TGGATGCTCTCATCAGGACAG \\
\hline & IL-6 & CTTCCAGCCAGTTGCCTTCTTG & GGTCTGTTGTGGGTGGTATCCTC \\
\hline & IFN- $\gamma$ & GCCACGGCACAGTCATTGA & TGCTGATGGCCTGATTGTCTT \\
\hline & $\mathrm{TNF}-\alpha$ & CCACCACGCTCTTCTGTCTACTG & GGGCTACGGGCTTGTCACTC \\
\hline & CYP1A1 & CAATGAGTTTGGGGAGGTTACTG & СССТTСТСАAАTGTCCTGTAGTG \\
\hline & TTP & CTCGGAGGACTTTGGAACAT & TGCAGTAGGCGAAGTAGGTG \\
\hline & GAPDH & TGAAGGTCGGTGTGAACGGATTTGG & ACGACATACTCAGCACCAGCATCAC \\
\hline
\end{tabular}

CYP1A1, cytochrome P450 1A1; IFN- $\gamma$, interferon- $\gamma$; IL, interleukin; TNF- $\alpha$, tumour necrosis factor- $\alpha$; TTP, tristetraprolin.

(1:20) antibodies overnight at $4^{\circ} \mathrm{C}$. Subsequently, Cy3-conjugated goat anti-mouse immunoglobulin (Ig)G (1:200) and fluorescein isothiocyanate-conjugated goat anti-rabbit $\operatorname{IgG}(1: 200)$ were used to stain the cells and sections, respectively. Finally, $1 \mathrm{mg} / \mathrm{ml}$ DAPI (Invitrogen; Thermo Fisher Scientific, Inc.) was used to stain the nuclei for total cell counting. The fluorescent signals were analysed by confocal laser scanning microscopy (Leica LAS AF Lite, version 2.8.0; Leica, Wetzlar, Germany), after the recording and merging of single-stained images.

RNA purification and RT-qPCR. Total RNA from intestinal mucosa and LoVo cells was extracted using TRIzol reagent (Invitrogen; Thermo Fisher Scientific, Inc.) according to the manufacturer's protocol. Spectrophotometry was used to determine the concentration of total RNA. For RT-qPCR, $1 \mu \mathrm{g}$ total RNA was converted to cDNA using a RT kit (Takara Bio, Inc., Otsu, Japan) in a $20 \mu \mathrm{l}$ volume according to the following temperature protocol: $37^{\circ} \mathrm{C}$ for $15 \mathrm{~min}, 85^{\circ} \mathrm{C}$ for $5 \mathrm{sec}$, followed by $5 \mathrm{~min}$ at $4^{\circ} \mathrm{C}$. Subsequently, SYBR-Green-based qPCR was used to measure the mRNA expression levels of CYP1A1, TTP, IFN- $\gamma$, TNF- $\alpha$, IL-1 $\beta$, IL- $6, \beta$-actin and GAPDH. The average quantification cycle $(\mathrm{Cq})$ value of triplicate wells with each primer set was calculated as the amount of gene product present in the sample. The relative mRNA expression levels were determined according to the ratio between the $\mathrm{Cq}$ value of the target gene and $\beta$-actin or GAPDH (28). The cycling conditions were as following: $94^{\circ} \mathrm{C}$ for $10 \mathrm{~min}, 40$ cycles at $94^{\circ} \mathrm{C}$ for $30 \mathrm{sec}, 60^{\circ} \mathrm{C}$ for $1 \mathrm{~min}$ and $68^{\circ} \mathrm{C}$ for $30 \mathrm{sec}$, followed by $7 \mathrm{~min}$ at $68^{\circ} \mathrm{C}$. The primer sequences were designed using online tools (https://www.ncbi. nlm.nih.gov/tools/primer-blast/) and are presented in Table I.

Western blot analysis. LoVo cells were washed three times with PBS and were lysed in cell lysis buffer (Intron Biotechnology, Inc., Seongnam, Korea) for $20 \mathrm{~min}$ on ice. The lysed cells were then centrifuged at $12,000 \mathrm{x} g$ for $10 \mathrm{~min}$ at $4^{\circ} \mathrm{C}$, and the supernatant was collected. The proteins were extracted from the intestinal mucosa using lysis buffer, followed by homogenization through sonication and the extraction mixture was centrifuged at $12,000 \mathrm{x}$ g for $15 \mathrm{~min}$ at $4^{\circ} \mathrm{C}$. The protein concentrations were measured using a bicinchoninic acid assay kit (Beyotime Institute of Biotechnology). Protein samples $(25 \mu \mathrm{g})$ were separated by $10 \%$ SDS-PAGE and the proteins were transferred to polyvinylidene fluoride membranes (Amersham; GEHealthcare, Chicago,IL,USA). The membranes were blocked in TBS containing $0.05 \%$ Tween-20 (TBS-T) and 5\% bovine serum albumin (BSA; Sigma-Aldrich, Inc.; Merck KGaA) for $2 \mathrm{~h}$ at $37^{\circ} \mathrm{C}$ and were then incubated with the following primary antibodies diluted in TBS-T containing 5\% BSA overnight at $4^{\circ} \mathrm{C}$ : Mouse anti-AhR (1:500), rabbit anti-CYP1A1 $(1: 1,000)$, rabbit anti-TTP $(1: 200)$, rabbit anti-MK2 $(1: 1,000)$, rabbit anti-p-MK2 $(1: 1,000)$ and rabbit anti-GAPDH $(1: 1,000)$. The membranes were washed three times in TBS-T, and were then incubated with goat anti-mouse and goat anti-rabbit horseradish peroxidase (HRP)-conjugated secondary antibodies $(1: 5,000)$, after which they were washed a further three times in TBS-T. Enhanced chemiluminescence (Millipore, Billerica, MA, USA) was used to detect the binding of HRP-conjugated antibodies according to the manufacturer's protocol.

Statistical analysis. Data are expressed as the mean \pm standard deviation. Differences among groups were assessed by analysis of variance using SPSS statistical software package (version 13.0; SPSS Inc., Chicago, IL, USA). The groups were compared using one-way analysis of variance (ANOVA) and Q-test. Comparisons between groups were conducted using paired t-tests. $\mathrm{P} \leq 0.05$ was considered to indicate a statistically significant difference.

\section{Results}

AhR deficiency results in increased colitis severity. Initially, the present study observed that administration of DSS produces more severe colitis in AhR-KO mice compared with in C57BL/6J 


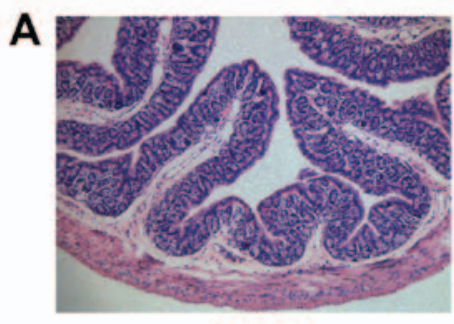

Sham

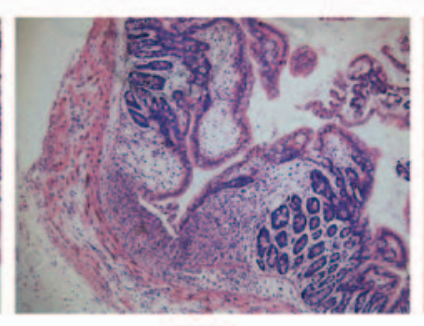

DSS

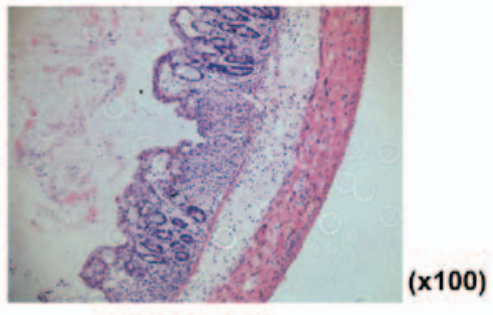

AhR-KO+DSS
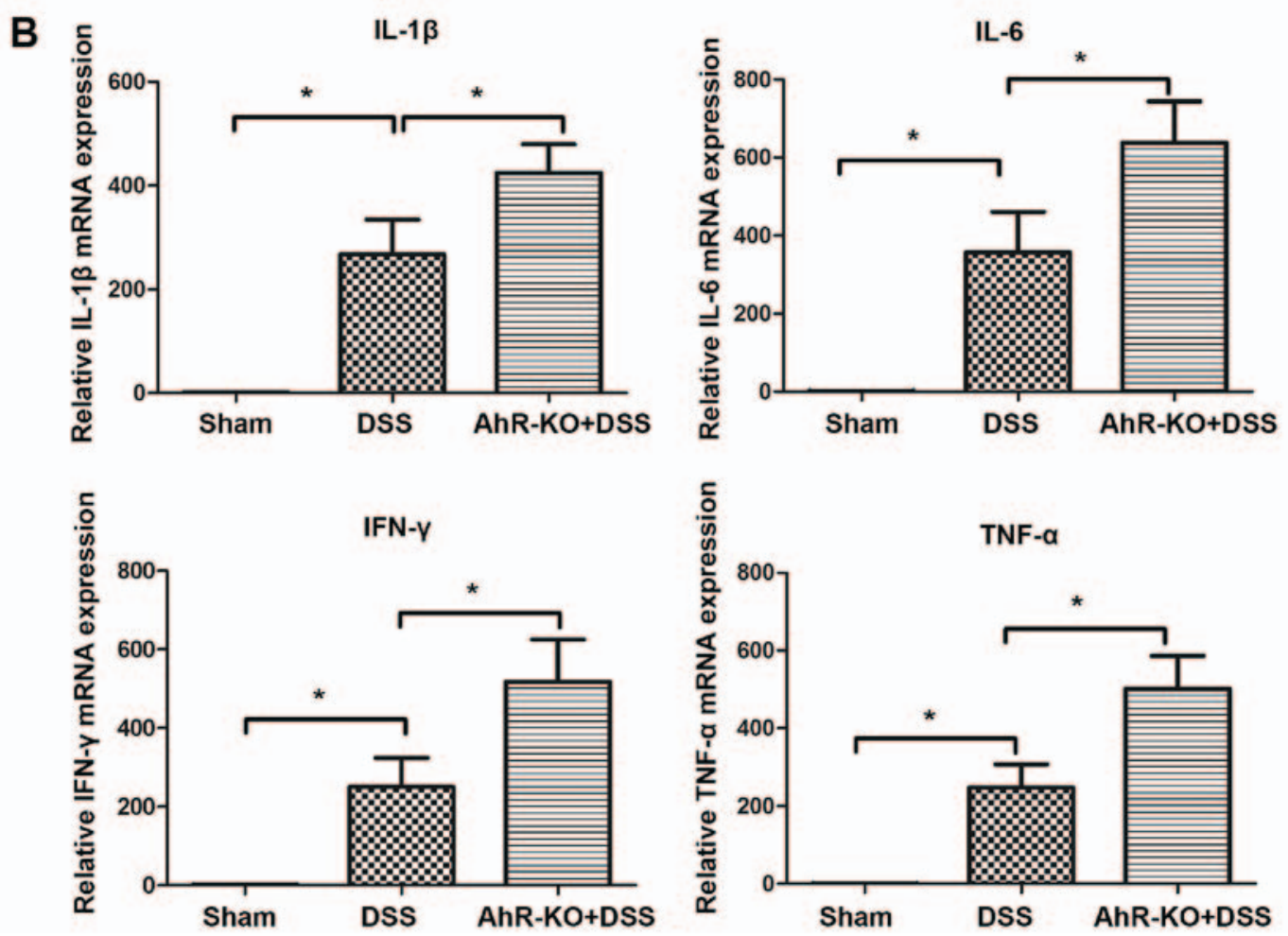

Figure 1. AhR deficiency results in increased colitis severity. AhR-KO mice and C57BL/6J WT mice were treated with 3\% DSS for 7 days. (A) Representative haematoxylin and eosin-stained colonic sections from DSS-treated C57BL/6J and AhR-KO mice (magnification, x100). (B) mRNA expression levels of IL-1 $\beta$, IL-6, IFN- $\gamma$ and TNF- $\alpha$ in colonic samples from mice treated as indicated in (A). mRNA expression was analysed by quantitative polymerase chain reaction. Data are presented as the mean \pm standard deviation of three experiments, in which samples from 3 mice/group were analysed. $\mathrm{P}<0.05$. AhR, aryl hydrocarbon receptor; DSS, dextran sulphate sodium; IFN- $\gamma$, interferon- $\gamma$; IL, interleukin; KO, knockout; TNF- $\alpha$, tumour necrosis factor- $\alpha$; WT, wild-type.

wild-type (WT) mice, as evidenced by histological examination of colonic tissue (Fig. 1A). Furthermore, mice in the AhR-KO group produced more inflammatory factors compared with those in the DSS-treated WT group. The mRNA expression levels of IL-1 $\beta$, IL- 6 , IFN- $\gamma$ and TNF- $\alpha$ were significantly increased in the AhR-KO + DSS group compared with in the DSS-treated WT group (Fig. 1B). These findings suggested that a deficiency in AhR may be associated with DSS-induced colitis.

AhR activation ameliorates DSS-induced colitis. To investigate whether AhR activation could attenuate experimental colitis, mice were administered FICZ for 5 days, beginning 2 days after the start of DSS administration (Fig. 2). DSS-treated mice exhibited significant weight loss 3-4 days after DSS treatment (Fig. 2B). On day 8 , mortality rate in the DSS group was $\sim 30 \%$ (Fig. 2C). In addition, colon length was decreased in DSS-treated mice (Fig. 2A). Conversely, DSS + FICZ-treated mice exhibited reduced weight loss and mortality ( $16.67 \%)$, and colon length was increased compared with in the DSS-treated mice (Fig. 2A-C). In addition, histological examination of colonic tissues from mice in the various groups revealed that mice treated with FICZ developed less severe colitis (Fig. 2E). The expression levels of IL-1 $\beta$, IL-6, IFN- $\gamma$ and TNF- $\alpha$ were significantly increased in colon samples from mice in the DSS-induced colitis group compared with in the vehicle-treated group (Fig. 2D). However, in mice treated with FICZ the mRNA expression levels of IFN- $\gamma$, IL-1 $\beta$, IL-6 and TNF- $\alpha$ were significantly reduced (Fig. 2D). These findings suggested that administration of FICZ may attenuate inflammation associated with DSS-induced colitis in mice, and that AhR has an important role in this attenuation.

FICZ upregulates expression of the RNA-binding protein TTP in mice with DSS-induced colitis. Recent studies have reported that TTP serves an important role in limiting inflammatory responses $(29,30)$. In addition, treatment of TTP-KO mice with DSS results in increased susceptibility to colitis (19). Furthermore, it has been demonstrated that TTP expression is decreased in $\mathrm{AhR}^{-/-}$cells (26). To explore whether TTP has 
A
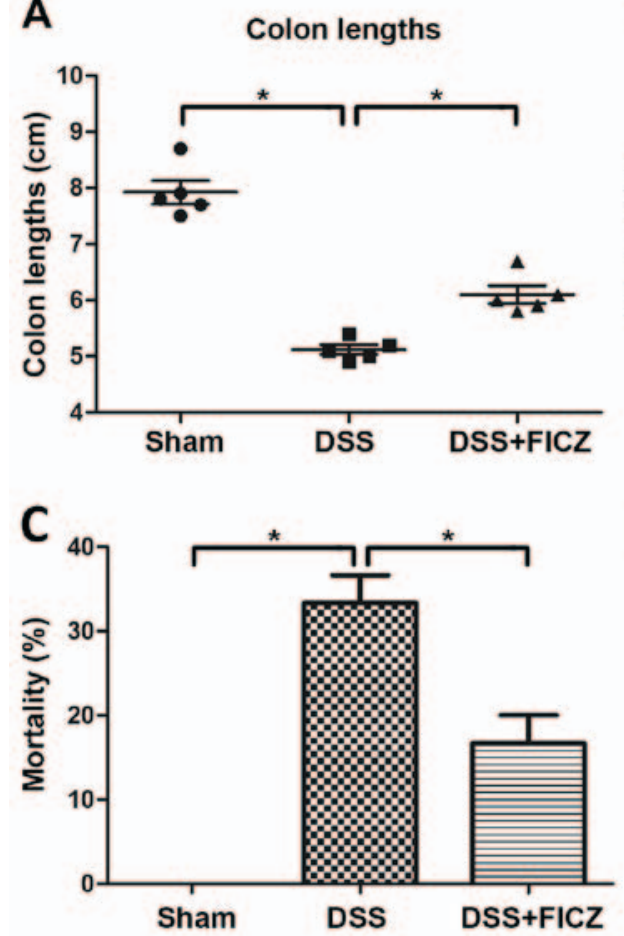

B

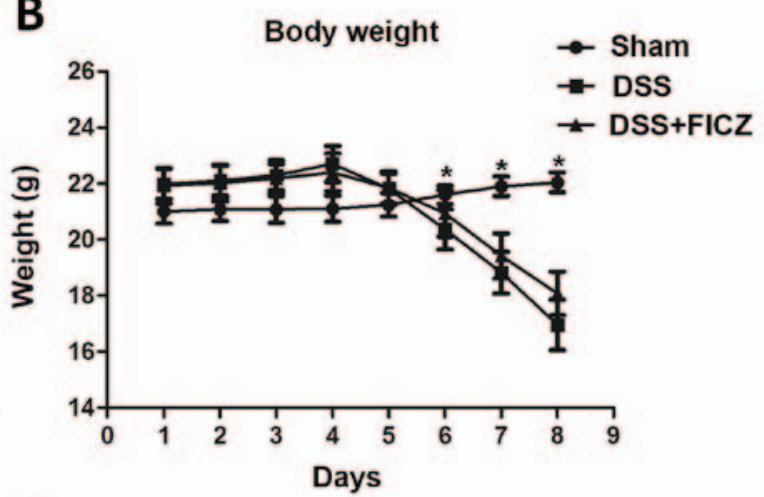

D

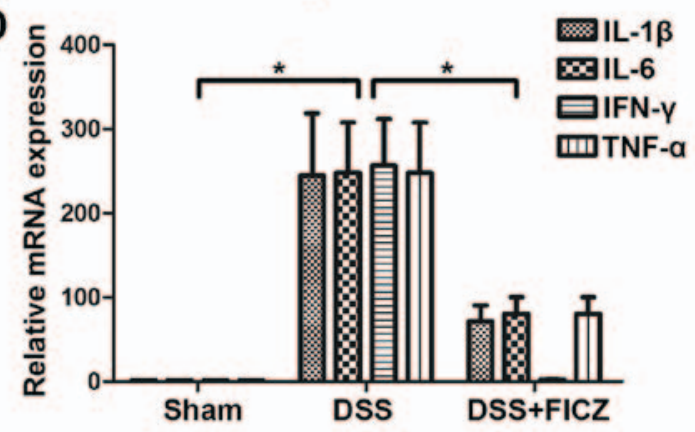

E

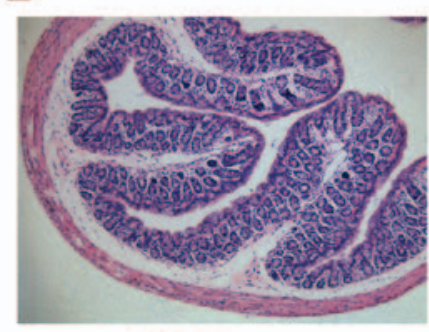

Sham

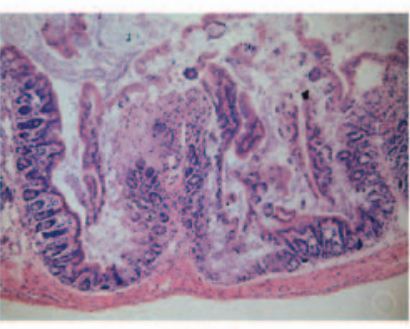

DSS

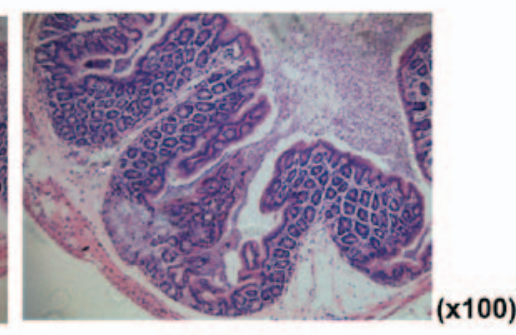

DSS+FICZ

Figure 2. AhR activation ameliorates DSS-induced colitis. (A) FICZ was intraperitoneally administered to mice for 5 days, beginning 2 days after the start of DSS administration. Colon lengths of mice in the sham, DSS and DSS + FICZ groups were measured on day 8. Data are presented as the mean \pm standard deviation of five experiments in which samples from 10 mice/group were analysed. (B) Changes in body weight were recorded daily. Weight data are presented as the cumulative mean \pm standard deviation obtained from five separate experiments. In each experiment, each group consisted of $\geq 10$ mice. (C) Percentage of mortality in the sham, DSS and DSS + FICZ groups was noted on day 8. Data are presented as the mean \pm standard deviation. (D) mRNA expression levels of IL-1 $\beta$, IL-6, IFN- $\gamma$ and TNF- $\alpha$ in colonic samples from mice treated as indicated in (A). mRNA expression was analysed by quantitative polymerase chain reaction. Data are presented as the mean \pm standard deviation of five experiments in which samples from 10 mice/group were analysed. (E) Representative haematoxylin and eosin-stained colonic sections of mice in the sham, DSS and DSS + FICZ groups on day 8 (magnification, x100). ${ }^{*} \mathrm{P}<0.05$ ( $\left.\mathrm{n}=10 / \mathrm{group}\right)$. AhR, aryl hydrocarbon receptor; DSS, dextran sulphate sodium; FICZ, 6-formylindolo(3,2-b)carbazole; IFN- $\gamma$, interferon- $\gamma$; IL, interleukin; KO, knockout; TNF- $\alpha$, tumour necrosis factor- $\alpha$; WT, wild-type.

a role in alleviating DSS-induced colitis, and to determine the association between TTP and AhR in this process, the expression levels of TTP were detected. The results indicated that the expression levels of AhR, CYP1A1 and TTP were downregulated in AhR-KO mice (Fig. 3A and B). Similarly, the expression levels of AhR and TTP were significantly decreased in mice with DSS-induced colitis (Fig. 3C and D).

The present study demonstrated that TTP expression was downregulated in AhR-KO mice. Therefore, in order to explore whether activation of AhR could upregulate the expression of TTP, mice were intraperitoneally injected with FICZ daily, beginning 2 days after the start of DSS administration. The expression levels of AhR, CYP1A1 and TTP were significantly increased following administration of FICZ to mice with DSS-induced colitis (Fig. 3C and D). Increased AhR, CYP1A1 and TTP expression was confirmed by western blotting (Fig. 3D). Furthermore, alterations in mRNA expression levels, as quantified by RT-qPCR, correlated with these protein alterations (Fig. 3C).

FICZ upregulates the RNA-binding protein TTP expression in an in vitro model. To further investigate the role of $\mathrm{AhR}$ and TTP in an in vitro model, LoVo human intestinal epithelial cells were treated with LPS $(10 \mu \mathrm{g} / \mathrm{ml})$ in the presence or absence of FICZ (100 nM) for $8 \mathrm{~h}$. The results demonstrated that IL-1 $\beta$ and IL-6 were significantly increased following treatment of the cells with LPS. However, the mRNA expression levels of IL-1 $\beta$ and IL-6 were significantly downregulated in the LPS + FICZ group compared with in the LPS group (Fig. 4A). This finding suggested that FICZ may inhibit LPS-induced inflammation in an in vitro model. Subsequently, the present study aimed to determine whether FICZ modulates the RNA-binding protein TTP. As illustrated in Fig. 4B and C, the protein and mRNA expression levels 

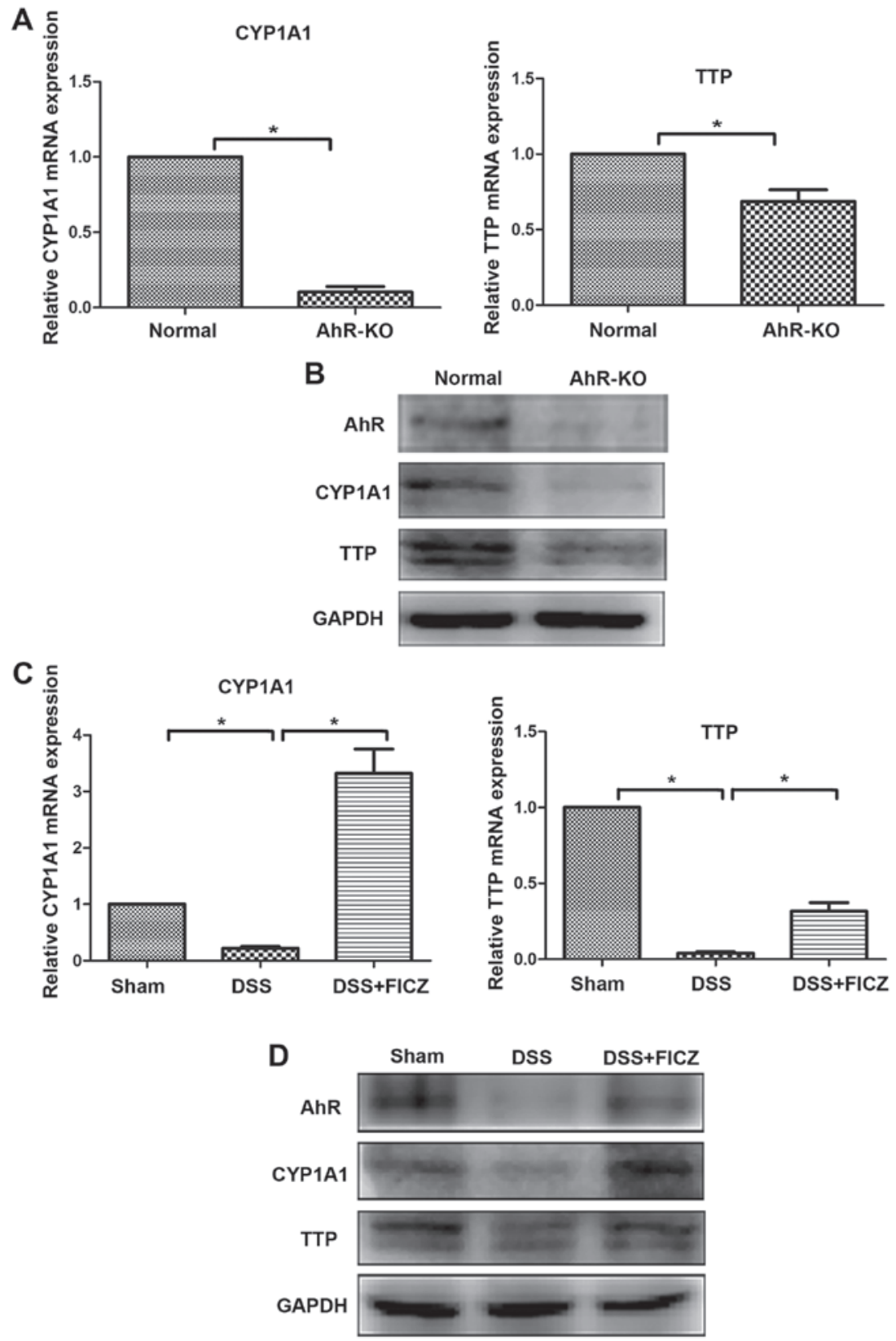

Figure 3. FICZ upregulates expression of the RNA-binding protein TTP in mice with DSS-induced colitis. (A) mRNA expression levels of CYP1A1 and TTP in colonic samples of WT and aryl hydrocarbon receptor (AhR) KO mice were determined by RT-qPCR. (B) Protein expression levels of AhR, CYP1A1 and TTP in colonic samples from WT and AhR-KO mice, as detected by western blotting. (C) FICZ was intraperitoneally administered to mice for 5 days, beginning 2 days after the start of DSS administration. The mRNA expression levels of CYP1A1 and TTP in colonic samples from the sham, DSS and DSS + FICZ groups were determined by RT-qPCR. (D) Mice were treated as indicated in (C), and the protein expression levels of AhR, CYP1A1 and TTP from colonic samples was detected by western blotting. Data are presented as the mean \pm standard deviation of five experiments in which samples from 10 mice/group were analysed. "P<0.05. AhR, aryl hydrocarbon receptor; CYP1A1, cytochrome P450 1A1; DSS, dextran sulphate sodium; FICZ, 6-formylindolo(3,2-b)carbazole; KO, knockout; RT-qPCR, reverse transcription-quantitative polymerase chain reaction; TTP, tristetraprolin; WT, wild-type.

of TTP were significantly increased in the LPS + FICZ group compared with in the LPS group. These results are in accordance with those obtained in the animal model. Unexpectedly, the protein expression levels of AhR were decreased following treatment with FICZ. This may be due to AhR degradation following FICZ treatment $(31,32)$. Immunofluorescence staining revealed that confluent LoVo cells treated with LPS and FICZ displayed an increased intensity of TTP staining compared with the LPS-treated group. In addition, the intensity of AhR staining was decreased in the LPS and FICZ-treated groups compared to the LPS-treated groups (Fig. 4D).
AhR regulates TTP expression through the $M K 2 / p-M K 2$ pathway. In order to explore the mechanism through which AhR regulates TTP, AhR expression was silenced in LoVo cells, after which the cells were treated with LPS and FICZ for $8 \mathrm{~h}$. As expected, the protein expression levels of AhR and TTP were decreased in cells in which AhR expression was silenced (Fig. 5A). Concurrently, TTP expression in AhR siRNA-transfected cells treated with LPS and FICZ was lower than that in normal cells treated with LPS and FICZ (Fig. 5B). These findings indicated that FICZ may upregulate TTP via AhR. In addition, LoVo cells were treated with LPS and FICZ, 


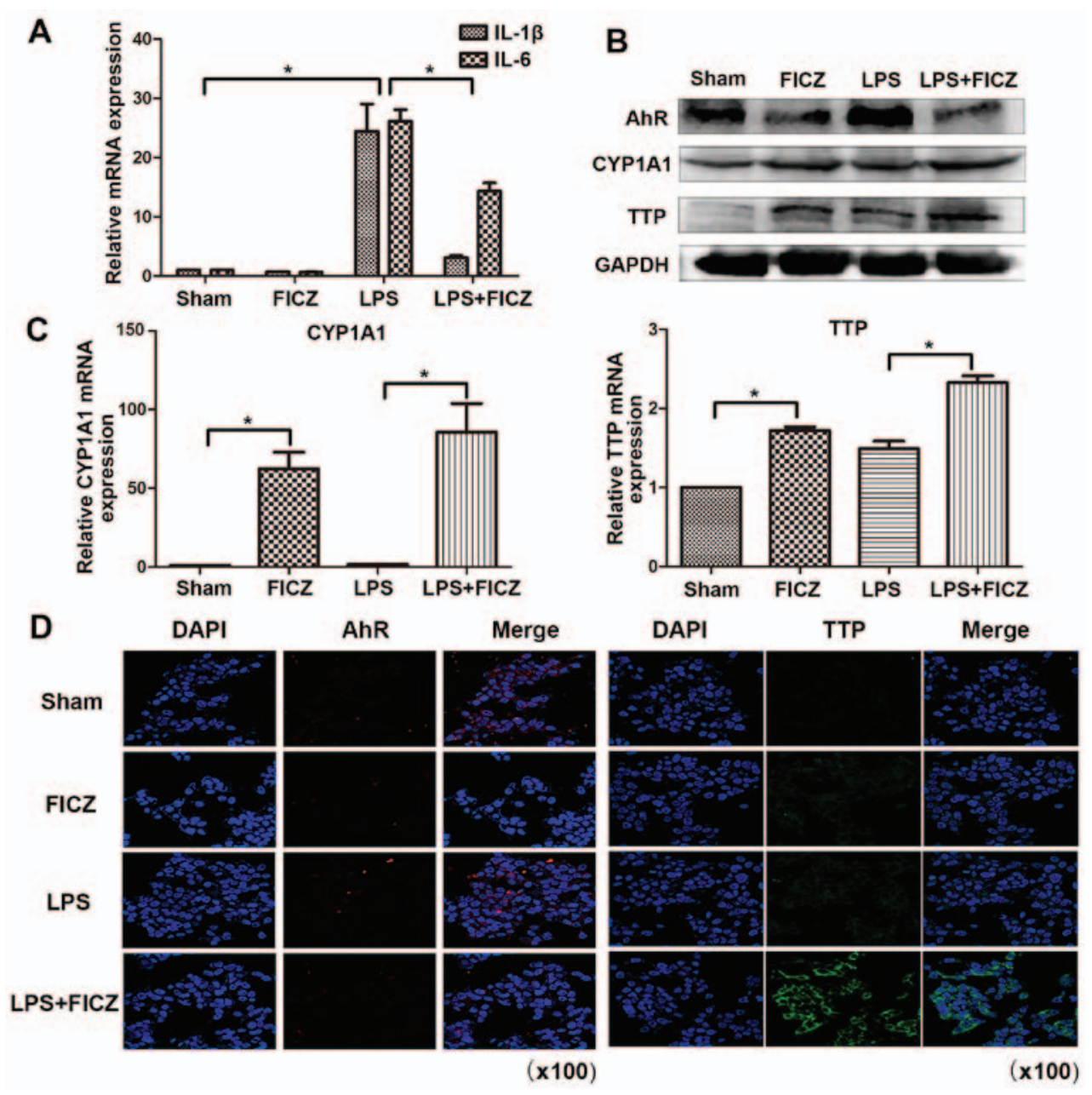

Figure 4. FICZ upregulates the RNA-binding protein TTP expression in an in vitro model. (A-D) LoVo human intestinal epithelial cells were treated with or without LPS $(10 \mu \mathrm{g} / \mathrm{ml})$ and FICZ $(100 \mathrm{nM})$ for $8 \mathrm{~h}$. The mRNA expression levels of (A) IL-1 $\beta$, IL-6, (C) CYP1A1 and TTP were determined by reverse transcription-quantitative polymerase chain reaction. (B) Protein expression levels of AhR, CYP1A1 and TTP were semi-quantified by western blotting. (D) Immunofluorescence was used to detect AhR and TTP expression. Red and green signals represent AhR and TTP, respectively (magnification, x100) Data are presented as the mean \pm standard deviation. " $\mathrm{P}<0.05$. AhR, aryl hydrocarbon receptor; CYP1A1, cytochrome P450 1A1; FICZ, 6-formylindolo(3,2-b) carbazole; IL, interleukin; TTP, tristetraprolin.

and the expression levels of MK2 and p-MK2 were measured, which are the classical upstream kinases of the TTP pathway, in order to determine whether AhR mediates TTP expression through the MK2/p-MK2 pathway. Notably, MK2 expression was not significantly altered; however, p-MK2 expression was reduced in the LPS + FICZ-treated group compared with in the LPS-treated group (Fig. 5C). Consistent with the expected results, these findings indicated that AhR may mediate TTP expression via the MK2/p-MK2 pathway. It should be noted that after AhR expression was silenced, MK2 expression was still not significantly altered; however, FICZ no longer downregulated p-MK2 expression in the LPS + FICZ-treated group (Fig. 5D). These findings suggested that AhR may serve a key role in the FICZ-mediated downregulation of p-MK2 expression.

\section{Discussion}

The present study demonstrated that mice deficient in AhR displayed increased susceptibility to colitis, and indicated that activation of AhR by FICZ could ameliorate DSS-induced colitis. In addition, AhR could inhibit the inflammation that occurs in DSS-induced colitis via the MK2/p-MK2/TTP pathway.
It has previously been reported that AhR serves an important role in IBD. AhR can inhibit inflammation and colitis in mice via the upregulation of IL-22 (11), and AhR activation significantly inhibited the production of IL- $1 \beta$, IL- 6 and TNF- $\alpha$ in LPS-treated dendritic cells (17), and attenuated LPS-induced inflammatory responses in SW480 human colon carcinoma cells (10). Furthermore, AhR can be activated by 2,3,7,8-tetrachlorodibenzo-p-dioxin and attenuates inflammation associated with CD (33). In addition, $\beta$-naphthoflavone, a nontoxic agonist of $\mathrm{AhR}$, has been reported to suppress the pathogenesis of DSS-induced colitis and attenuate DSS-induced colitis (10). These findings suggested that AhR expression in intestinal epithelial cells is involved in the prevention of colitis.

The present study examined the expression and functional role of AhR in a mouse model of DSS-induced colitis. The results indicated that DSS induces more severe colitis in AhR-KO mice compared with in wild-type mice. This finding suggested that a deficiency in AhR may result in a decrease in resistance to inflammation. In addition, the present study assessed whether activation of AhR by FICZ could attenuate DSS-induced colitis. Treatment of mice with FICZ for 5 days, starting 2 days after the initiation of DSS administration, 

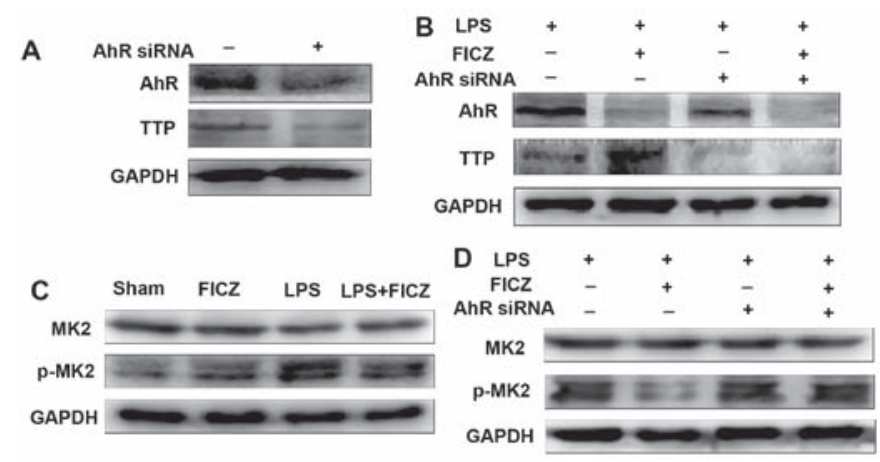

Figure 5. AhR regulates TTP expression via the MK2/p-MK2 pathway. (A) Confluent LoVo cells were transfected with AhR siRNA for $48 \mathrm{~h}$. Protein expression levels of AhR and TTP were detected by western blotting. (B) Cells were transfected with AhR siRNA, and were treated with or without LPS $(10 \mu \mathrm{g} / \mathrm{ml})$ and FICZ $(100 \mathrm{nM})$ for $8 \mathrm{~h}$; western blotting was used to detect the protein expression levels of AhR and TTP. (C) LoVo human intestinal epithelial cells were treated with or without LPS $(10 \mu \mathrm{g} / \mathrm{ml})$ and FICZ (100 nM) for $8 \mathrm{~h}$. Protein expression levels of MK2 and p-MK2 were detected by western blotting. (D) Cells were treated as indicated in $(\mathrm{C})$ and were transfected with AhR siRNA; western blotting was used to detect the protein expression levels of MK2 and p-MK2. AhR, aryl hydrocarbon receptor; FICZ, 6-formylindolo(3,2-b)carbazole; LPS, lipopolysaccharide; MK2, mitogen-activated protein kinase-activated protein kinase 2; p-MK2, phosphorylated-MK2; siRNA, small interfering RNA: TTP, tristetraprolin.

significantly inhibited the expression of inflammatory factors. Based on these findings, it may be suggested that AhR activation attenuates DSS-induced colitis.

The main aetiology of IBD is thought to be associated with disordered immune function, in which the organism releases a mass of inflammatory factors, resulting in an imbalance between pro- and anti-inflammatory factors, which in turn disrupts intestinal barrier function. In recent years, the mechanisms through which factors that mediate the stability of mRNAs coding inflammatory factors exert their anti-inflammatory effects have attracted more attention. RNA-binding proteins, which may bind to AREs located in the 3'-UTRs of target mRNAs and direct them to exosomes for rapid degradation $(34,35)$, can reduce the expression of various inflammatory factors. RNA-binding proteins, including TTP and HuR, are found in numerous protein families. It has previously been reported that TTP-KO mice display more severe autoimmune dysfunction, inflammatory arthritis and UC compared with their WT counterparts (20). Furthermore, in $\mathrm{AhR}^{-/-}$cells, the expression of TTP is downregulated (26). AhR also inhibits inflammation via the downregulation of another RNA-binding protein, HuR (27). These findings suggested that AhR activation may regulate activity of the RNA-binding protein TTP.

The present study assessed whether AhR could regulate TTP expression in the gut and investigated the role of TTP in IBD. Initially, the results demonstrated that TTP expression was decreased in DSS-treated mice compared with in vehicle-treated animals. In addition, consistent with results reported in the literature (26), the expression levels of AhR and TTP were downregulated in AhR-KO mice. When DSS-treated mice were treated with FICZ, TTP expression was upregulated. These findings suggested that TTP expression may be associated with IBD and that FICZ, which activates the AhR pathway, may exert its anti-inflammatory effects by upregulating the RNA-binding protein TTP.
Although AhR mediates the expression of TTP, the mechanism underlying this effect remains unknown. It has been suggested that phosphorylation of MK2, which occurs upstream of TTP, modulates the activity and expression of TTP. MK2 can phosphorylate the TTP protein (36.37). In addition, the stability, expression and function of TTP are reported to be regulated by the MK2/p-MK2 pathway $(36,38,39)$. Activation of the MK2/p-MK2 pathway has been reported to abolish TTP-mediated suppression of IL-6 3'-UTR reporter activity (40). In addition, it has been demonstrated that the MK2/p-MK2 cascade is involved in regulating the stability of ARE-containing mRNAs (41). Recent studies have clearly indicated that the MK2/p-MK2 signalling cascade can regulate TTP-mediated mRNA stability of IL-6 and TNF- $\alpha$ (42).

In the present study, following treatment with FICZ, MK2 expression was not significantly altered; however, the expression of p-MK2 was downregulated. Conversely, when AhR expression was silenced, FICZ no longer downregulated p-MK2 expression. These findings suggested that FICZ upregulates the expression of TTP by downregulating $\mathrm{p}-\mathrm{MK} 2$ expression.

In conclusion, the present study demonstrated that AhR serves an important role in attenuating DSS-induced colitis. AhR-mediated protection operates in part by inducing TTP via the MK2/p-MK2 pathway. The present study is the first, to the best of our knowledge, to demonstrate a dependence on TTP for AhR-mediated protection against DSS-induced colitis. Since AhR may serve important roles in protection against DSS-induced colitis, the AhR-TTP pathway may be considered an attractive candidate for the treatment of UC.

\section{Acknowledgements}

The present study was supported by grants from the National Natural Science Foundation of China (grant nos. NSFC 81330013 and NSFC 81272078 to H.Y., and NSFC81300275 to L.S.) and the Program of Changjiang Scholars and Innovative Research (grant no. IRT 13050 to H.Y.).

\section{References}

1. Bamias G, Martin C III, Mishina M, Ross WG, Rivera-Nieves J, Marini M and Cominelli F: Proinflammatory effects of TH2 cytokines in a murine model of chronic small intestinal inflammation. Gastroenterology 128: 654-666, 2005.

2. Fiocchi C: Inflammatory bowel disease: Etiology and pathogenesis. Gastroenterology 115: 182-205, 1998.

3. Strober W, Fuss IJ and Blumberg RS: The immunology of mucosal models of inflammation. Annu Rev Immunol 20: 495-549, 2002.

4. Uhlig HH and Powrie F: The role of mucosal Tlymphocytes in regulating intestinal inflammation. Springer Semin Immunopathol 27: 167-180, 2005.

5. Kaser A, Zeissig S and Blumberg RS: Inflammatory bowel disease. Annu Rev Immunol 28: 573-621, 2010.

6. Targan SR: Hanauer Sb, Vandeventer SH, Lloydmayer and Present D: A short-term study of chimeric monoclonal antibody CA2 to tumor necrosis factor a for Crohn's disease. N Engl J Med 337: 1029-1036, 1997.

7. Baumgart DC and Sandborn WJ: Inflammatory bowel disease: Clinical aspects and established and evolving therapies. Lancet 369: 1641-1657, 2007.

8. Neurath MF, Fuss I, Kelsall BL, Stüber E and Strober W: Antibodies to interleukin 12 abrogate established experimental colitis in mice. J Exp Med 182: 1281-1290, 1995.

9. Ito H, Takazoe M, Fukuda Y, Hibi T, Kusugami K, Andoh A, Matsumoto T, Yamamura T, Azuma J and Nishimoto N: A pilot randomized trial of a human anti-interleukin- 6 receptor monoclonal antibody in active Crohn's disease. Gastroenterology 126: 989-996, discussion 947, 2004. 
10. Furumatsu K, Nishiumi S, Kawano Y, Ooi M, Yoshie T, Shiomi Y, Kutsumi H, Ashida H, Fujii-Kuriyama Y, Azuma T, et al: A role of the aryl hydrocarbon receptor in attenuation of colitis. Dig Dis Sci 56: 2532-2544, 2011.

11. Monteleone I, Rizzo A, Sarra M, Sica G, Sileri P, Biancone L, MacDonald TT, Pallone F, Monteleone G: Aryl hydrocarbon receptor-induced signals upregulate IL-22 production and inhibit inflammation in the gastrointestinal tract. Gastroenterology 141: 237-248.e1, 2011.

12. Ji T, Xu C, Sun L, Yu M, Peng K, Qiu Y, Xiao W and Yang H: Aryl hydrocarbon receptor activation downregulates IL-7 and reduces inflammation in a mouse model of DSS-induced colitis. Dig Dis Sci 60: 1958-1966, 2015.

13. Denis M, Cuthill S, Wikström AC, Poellinger L and Gustafsson JA Association of the dioxin receptor with the $\mathrm{Mr} 90,000$ heat shock protein: A structural kinship with the glucocorticoid receptor. Biochem Biophys Res Commun 155: 801-807, 1988.

14. Kewley RJ, Whitelaw ML and Chapman-Smith A: The mammalian basic helix-loop-helix/PAS family of transcriptional regulators. Int J Biochem Cell Biol 36: 189-204, 2004

15. SogawaK andFujii-Kuriyama Y: Ahreceptor, anovelligand-activated transcription factor. J Biochem 122: 1075-1079, 1997.

16. Lee YH, Lin CH, Hsu PC, Sun YY, Huang YJ, Zhuo JH, Wang CY, Gan YL, Hung CC, Kuan CY, et al: Aryl hydrocarbon receptor mediates both proinflammatory and anti-inflammatory effects in lipopolysaccharide-activated microglia. Glia 63: 1138-1154, 2015.

17. Wang C, Ye Z, Kijlstra A, Zhou Y and Yang P: Activation of the aryl hydrocarbon receptor affects activation and function of human monocyte-derived dendritic cells. Clin Exp Immunol 177: 521-530, 2014

18. Barouki R, Coumoul X and Fernandez-Salguero PM: The aryl hydrocarbon receptor, more than a xenobiotic-interacting protein. FEBS Lett 581: 3608-3615, 2007.

19. Joe Y, Uddin MJ, Zheng M, Kim HJ, Chen Y, Yoon NA, Cho GJ, Park JW and Chung HT: Tristetraprolin mediates anti-inflammatory effect of carbon monoxide against DSS-induced colitis. PLoS One 9: e88776, 2014.

20. Sanduja S, Blanco FF, Young LE, Kaza V and Dixon DA: The role of tristetraprolin in cancer and inflammation. Front Biosci (Landmark Ed) 17: 174-188, 2012

21. Fan XC and Steitz JA: Overexpression of HuR, a nuclear-cytoplasmic shuttling protein, increases the in vivo stability of ARE-containing mRNAs. EMBO J 17: 3448-3460, 1998.

22. Carballo E, Lai WS and Blackshear PJ: Feedback inhibition of macrophage tumor necrosis factor-alpha production by tristetraprolin. Science 281: 1001-1005, 1998

23. Kratochvill F, Machacek C, Vogl C, Ebner F, Sedlyarov V, Gruber AR, Hartweger H, Vielnascher R, Karaghiosoff M, Rülicke T, et al: Tristetraprolin-driven regulatory circuit controls quality and timing of mRNA decay in inflammation. Mol Syst Biol 7: 560, 2011.

24. Molle C, Zhang T, Ysebrant de Lendonck L, Gueydan C, Andrianne M, Sherer F, Van Simaeys G, Blackshear PJ, Leo O and Goriely S: Tristetraprolin regulation of interleukin 23 mRNA stability prevents a spontaneous inflammatory disease. J Exp Med 210: 1675-1684, 2013.

25. Taylor GA, Carballo E, Lee DM,Lai WS, Thompson MJ,Patel DD, Schenkman DI, Gilkeson GS, Broxmeyer HE, Haynes BF, et al: A pathogenetic role for TNF alpha in the syndrome of cachexia, arthritis, and autoimmunity resulting from tristetraprolin (TTP) deficiency. Immunity 4: 445-454, 1996.

26. Chang X, Fan Y, Karyala S, Schwemberger S, Tomlinson CR, Sartor MA and Puga A: Ligand-independent regulation of transforming growth factor betal expression and cell cycle progression by the aryl hydrocarbon receptor. Mol Cell Biol 27: 6127-6139, 2007.

27. Zago M, Sheridan JA, Nair P, Rico de Souza A, Gallouzi IE, Rousseau S, Di Marco S, Hamid Q, Eidelman DH and Baglole CJ: Aryl hydrocarbon receptor-dependent retention of nuclear HuR suppresses cigarette smoke-induced cyclooxygenase-2 expression independent of DNA-binding. PLoS One 8: e74953, 2013.

28. Livak KJ and Schmittgen TD: Analysis of relative gene expression data using real-time quantitative PCR and the 2(-Delta Delta C(T)) Method. Methods 25: 402-408, 2001
29. Kratochvill F, Gratz N, Qualls JE, Van De Velde LA, Chi H, Kovarik P and Murray PJ: Tristetraprolin limits inflammatory cytokine production in tumor-associated macrophages in an mRNA decay-independent manner. Cancer Res 75: 3054-3064, 2015.

30. Ross EA, Smallie T, Ding Q, O'Neil JD, Cunliffe HE, Tang T, Rosner DR, Klevernic I, Morrice NA, Monaco C, et al: Dominant suppression of inflammation via targeted mutation of the mRNA destabilizing protein tristetraprolin. J Immunol 195: 265-276, 2015.

31. Song Z and Pollenz RS: Ligand-dependent and independent modulation of aryl hydrocarbon receptor localization, degradation, and gene regulation. Mol Pharmacol 62: 806-816, 2002.

32. Pollenz RS: The mechanism of AH receptor protein downregulation (degradation) and its impact on $\mathrm{AH}$ receptor-mediated gene regulation. Chem Biol Interact 141: 41-61, 2002.

33. Benson JM and Shepherd DM: Aryl hydrocarbon receptor activation by TCDD reduces inflammation associated with Crohn's disease. Toxicol Sci 120: 68-78, 2011.

34. Lai WS, Carballo E, Strum JR, Kennington EA, Phillips RS and Blackshear PJ: Evidence that tristetraprolin binds to AU-rich elements and promotes the deadenylation and destabilization of tumor necrosis factor alpha mRNA. Mol Cell Biol 19: 4311-4323, 1999.

35. Chen CY, Gherzi R, Ong SE, Chan EL, Raijmakers R, Pruijn GJ, Stoecklin G, Moroni C, Mann M and Karin M: AU binding proteins recruit the exosome to degrade ARE-containing mRNAs. Cell 107: 451-464, 2001.

36. Mahtani KR, Brook M, Dean JLE, Sully G, Saklatvala J and Clark AR: Mitogen-activated protein kinase p38 controls the expression and posttranslational modification of tristetraprolin, a regulator of tumor necrosis factor alpha mRNA stability. Mol Cell Biol 21: 6461-6469, 2001.

37. Chrestensen CA, Schroeder MJ, Shabanowitz J, Hunt DF, Pelo JW, Worthington MT and Sturgill TW: MAPKAP kinase 2 phosphorylates tristetraprolin on in vivo sites including Ser178, a site required for 14-3-3 binding. J Biol Chem 279: 10176-10184, 2004.

38. Brook M, Tchen CR, Santalucia T, McIlrath J, Arthur JS, Saklatvala $J$ and Clark AR: Posttranslational regulation of tristetraprolin subcellular localization and protein stability by $\mathrm{p} 38$ mitogen-activated protein kinase and extracellular signal-regulated kinase pathways. Mol Cell Biol 26: 2408-2418, 2006.

39. Hitti E, Iakovleva T, Brook M, Deppenmeier S, Gruber AD, Radzioch D, Clark AR, Blackshear PJ, Kotlyarov A and Gaestel M: Mitogen-activated protein kinase-activated protein kinase 2 regulates tumor necrosis factor mRNA stability and translation mainly by altering tristetraprolin expression, stability, and binding to adenine/uridine-rich element. Mol Cell Biol 26: 2399-2407, 2006

40. Zhao W, Liu M, D'Silva NJ and Kirkwood KL: Tristetraprolin regulates interleukin-6 expression through p38 MAPK-dependent affinity changes with mRNA 3' untranslated region. J Interferon Cytokine Res 31: 629-637, 2011.

41. Sun L, Stoecklin G, Van Way S, Hinkovska-Galcheva V, Guo RF, Anderson P and Shanley TP: Tristetraprolin (TTP)-14-3-3 complex formation protects TTP from dephosphorylation by protein phosphatase $2 \mathrm{a}$ and stabilizes tumor necrosis factor-alpha mRNA. J Biol Chem 282: 3766-3777, 2007.

42. O'Dea KP, Dokpesi JO, Tatham KC, Wilson MR and Takata M: Regulation of monocyte subset proinflammatory responses within the lung microvasculature by the p38 MAPK/MK2 pathway. Am J Physiol Lung Cell Mol Physiol 301: L812-L821, 2011.

This work is licensed under a Creative Commons

Attribution-NonCommercial-NoDerivatives 4.0 International (CC BY-NC-ND 4.0) License. 